$05,11,12$

\title{
Фазовый переход в цилиндрических изинговых нанопроволоках и нанотрубках: приближение теории молекулярного поля
}

\author{
() В.А. Танрывердиев, В.С. Тагиев, М.Н. Абдуллаев, Г.Г. Керимова \\ Институт фозики НАНА, \\ Баку, Азербайджан \\ E-mail: vahid_tanriverdi@yahoo.com \\ (Поступила в Редакцию 13 августа 2018 г. \\ В окончательной редакции 7 ноября 2018 г. \\ Принята к публикации 7 ноября 2018 г.)
}

В рамках приближения теории молекулярного поля было изучено критическое поведение цилиндрических Изинговых нанопроволок и нанотрубок. Рассматриваемая, в данной работе, модель состоит из ферромагнитных спинов $S_{c}$, находящихся в сердцевине системы и ферромагнитных спинов $S_{s}$, находящихся на поверхностной стенке, которые связаны между собой обменной связью $J_{1}$. Температура перехода $T_{c}$ для таких систем вычислена, как функция параметров обменного взаимодействия. Исследовано влияние поверхностного обменного взаимодействия и поверхностной связи на магнитодинамическое поведение системы. Некоторые характеристические свойства, полученные на фазовой диаграмме, связаны с отношением характерных физических параметров на поверхности и сердцевине вышеприведенных наноструктур.

DOI: 10.21883/FTT.2019.04.47414.233

\section{1. Введение}

Сравнительно с недавнего времени значительное внимание стало уделяться изучению магнитных свойств наноструктурных материалов, таких как нанотрубки, нанопроволока, наноконусы, нанокольца и т.д. [1-3]. Их магнитные, так и электрические свойства сильно отличаются от подобных им объемных материалов, а их физические свойства очень сильно подвержены влиянию размерных эффектов и геометрической конфигурации материала [4-6]. Эти факты дают сильную мотивацию для дальнейших активных исследований наномагнитных структур. С другой стороны, магнитные наноструктуры являются объектами большого исследовательского интереса, как в экспериментальном, так и в теоретическом аспекте благодаря их возможному применению в нанобиологии и медицине, для получения магниторезонансной картины микробиологических изучаемых объектов, и как средство доставки лекарственных препаратов до клетки, избирательного теплового и электрического воздействия на них $[7,8]$. Важную роль, данные наносистемы, также играют в создании сверхчувствительных датчиков [9-12].

Недавние достижения в микропроцессорной технологии представили возможность получить магнитные материалы с различными типами структур и размеров в нанометровом диапазоне. Экспериментально, магнитные свойства магнитных наноматериалов были охарактеризованы мессбауровскими измерениями, измерениями температурной и полевой зависимости намагниченности, ферромагнитным резонансом и ЭПР [13,14]. На ряду с этим активно разрабатываются технологии получения качественных магнитных нанопроволок таких, как $\mathrm{Fe}-\mathrm{Co}[15], \mathrm{Co}-\mathrm{Pt}[16], \mathrm{Ni}-\mathrm{Fe}$ [17], $\mathrm{Fe}_{3} \mathrm{O}_{4}$ [18],
$\mathrm{Fe}-\mathrm{Pt}$ [19]. C другой стороны также успешно были изготовлены и исследованы, различными способами, такие магнитные нанотрубки, как $\mathrm{Fe}-\mathrm{Pt}$ и $\mathrm{Fe}_{3} \mathrm{O}_{4}$ [20].

Современные теорфизические методы, такие как гриновские функции (ФГ), теория среднего поля, метод эффективного поля, с учетом корреляций (МЭП), метод Монте-Карло (МК), трансферматричный формализм, техника рекуррентных соотношений, широко используются в изучении таких нестандартных систем [21-24].

В этой статье, методом приближения молекулярного поля, исследуется фазовый переход в цилиндрической нанопроволоке и нанотрубке, состоящих из двух магнитных стенок (внутренней - сердцевины и внешней поверхностной), описывающиеся моделью Изинга.

В разделе 2 схематически представлена рассматриваемая модель и описывающий ее гамильтониан и получены уравнения, которые определяют температуру переходов. Полученные уравнения носят общий характер для произвольных обменных взаимодействий. Численные результаты и обсуждение даются в разделе 3.

\section{2. Модель и формализм}

На рис. 1 представлена нанопроволока и нанотрубка, которые состоят из поверхностной и внутренней (сердцевина) атомных стенок. Сердцевина окружена поверхностной стенкой.

Магнитные атомы, находящиеся на поверхностной стенке, связаны обменной связью $J_{1}$ с атомами находящимися в сердцевине. Белые и черные точки на рис. 1 есть магнитные атомы образующие сердцевину и поверхностную стенку соответственно. Линии, соединяю- 

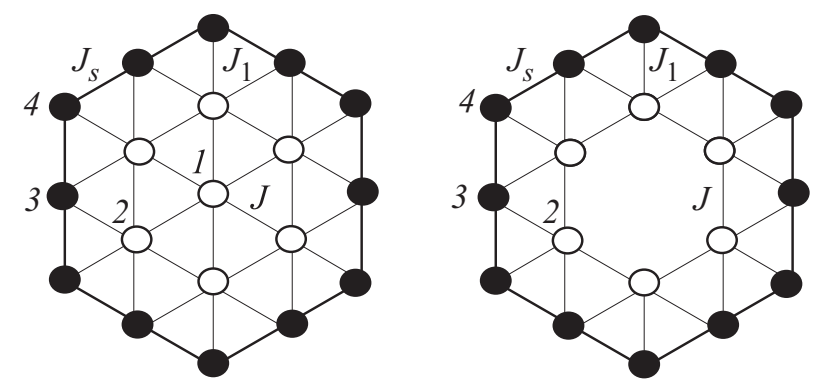

Рис. 1. Схематическое представление цилиндрических нанопроволок $(a)$ и нанотрубок $(b)$. Белыми и черными кружками отмечены магнитные атомы образующие сердцевину и поверхностную стенку соответственно.

щие их, представляют собой, схематично, наиближайшее соседнее обменное взаимодействие. С другой стороны, каждый Изинговский спин связан с наиближайшими спинами соседних слоев вдоль нанотрубки.

Гамильтониан системы может быть записан в следующем форме:

$$
H=-J_{s} \sum_{\langle i, j\rangle} S_{i}^{z} S_{j}^{z}-J \sum_{\langle m, n\rangle} S_{m}^{z} S_{n}^{z}-J_{1} \sum_{\langle i, m\rangle} S_{i}^{z} S_{n}^{z}
$$

$\langle i, j\rangle,\langle m, n\rangle$ и $(i, m)$ отмечена сумма по всем парам соседних спинов на поверхности и сердцевине, а также совместно на поверхности и сердцевине соответственно. $J_{s}$ есть обменное взаимодействие между двумя атомами, находящимся на поверхностной стенке, а $J$ - обменное взаимодействие в самой сердцевине. Для того чтобы выяснить влияние поверхностных эффектов на физические свойства системы, введем поверхностное обменное взаимодействие в следующем виде $J_{s}=J\left(1+\Delta_{s}\right)$. Для простоты вычислений, здесь мы пренебрегаем внешним магнитным полем. Ось $z$-координатной системы направлена вдоль основной оси рассматриваемых нанообъектов. В расчет также принимается симметрия нанопроволоки, где различают спины четырех типов рис. 1, $a$ и спины трех типов для нанотрубки рис. $1, b$, так два спина принадлежащих сердцевине (отмеченные, как 1 и 2) и два поверхностных спина (отмеченные, как 3 и 4).

Согласно модели молекулярного поля средний спин $m_{\tau}=\left\langle S_{\tau}^{z}\right\rangle \quad(\tau=1,2,3,4$ для нанопроволоки и $\tau=2,3,4$ для нанотрубки) определяется из следующих установленных уравнений [14]

$$
\left\{\begin{array}{l}
m_{1}=B_{S}\left[\left(6 J m_{2}+2 J m_{1}\right) / k T\right] \\
m_{2}=B_{S}\left[\left(J m_{1}+4 J m_{2}+2 J_{1} m_{3}+J_{1} m_{4}\right) / k T\right] \\
m_{3}=B_{S}\left[\left(2 J_{1} m_{2}+2 J_{s} m_{3}+2 J_{s} m_{4}\right) / k T\right] \\
m_{4}=B_{S}\left[\left(J_{1} m_{2}+2 J_{s} m_{3}+2 J_{s} m_{4}\right) / k T\right]
\end{array}\right.
$$

для нанопроволоки и

$$
\left\{\begin{array}{l}
m_{2}=B_{S}\left[\left(4 J m_{2}+2 J_{1} m_{3}+J_{1} m_{4}\right) / k T\right] \\
m_{3}=B_{S}\left[\left(2 J_{1} m_{2}+2 J_{s} m_{3}+2 J_{s} m_{4}\right) / k T\right] \\
m_{4}=B_{S}\left[\left(J_{1} m_{2}+2 J_{s} m_{3}+2 J_{s} m_{4}\right) / k T\right]
\end{array}\right.
$$

для нанотрубок. Здесь $k-$ есть Больцмановская константа. $B_{S}$ - есть брюльеновская функция:

$$
B_{S}(x)=\frac{2 S+1}{2 S} \operatorname{coth}\left(\frac{2 S+1}{2 S} x\right)-\frac{1}{2 S} \operatorname{coth}\left(\frac{1}{2 S} x\right) .
$$

Когда температура системы становится выше критической $T_{c}$, вся система становится размагниченной и средняя атомная намагниченность приближается к нулю. Около критической температуры $T_{c}$ средний спин $m_{\tau}$ становится малым. Для малых аргументов всеми членами порядка выше, чем линейные, можно пренебречь и выражение (4) можно записать в следующем виде:

$$
B_{S}(x) \approx \frac{S+1}{3 S} x .
$$

Для получения критической температуры, мы рассмотрим только линейные члены намагниченности и получим уравнение в матричном виде:

— для нанопроволок

$$
A m \equiv\left(\begin{array}{cccc}
\frac{3 S_{c} k T}{\left(S_{c}+1\right)-2 J} & -6 J & 0 & 0 \\
-J & \frac{3 S_{c} k T}{\left(S_{c}+1\right)-4 J} & -2 J_{1} & -J_{1} \\
0 & -2 J_{1} & \frac{3 S_{s} k T}{\left(S_{s}+1\right)-2 J_{s}} & -2 J_{s} \\
0 & -J_{1} & -2 J_{s} & \frac{3 S_{s} k T}{\left(S_{s}+1\right)-2 J_{s}}
\end{array}\right) \cdot\left(\begin{array}{l}
m_{1} \\
m_{2} \\
m_{3} \\
m_{4}
\end{array}\right)=0
$$

— для нанотрубок

$$
A m \equiv\left(\begin{array}{ccc}
\frac{3 S_{c} k T}{\left(S_{c}+1\right)-4 J} & -2 J_{1} & -J_{1} \\
-2 J_{1} & \frac{3 S_{s} k T}{\left(S_{s}+1\right)-2 J_{s}} & -2 J_{s} \\
-J_{1} & -2 J_{s} & \frac{3 S_{s} k T}{\left(S_{s}+1\right)-2 J_{s}}
\end{array}\right) \cdot\left(\begin{array}{l}
m_{2} \\
m_{3} \\
m_{4}
\end{array}\right)=0 .
$$

Температура перехода для каждой системы определяется из соотношения $\operatorname{Det} A=0$.

\section{3. Обсуждение полученных результатов}

В этом разделе рассмотрим некоторые типичные фазовые диаграммы в рассматриваемых двух наносистемах, используя формулировку, приведенную в разделе 2. Приведенный здесь формализм температуры перехода является универсальным и может быть использован в получении различных параметров, характеризующих систему. Также наши вычисления применимы, как для ферромагнитного, так и для антиферромагнитного случаев. 


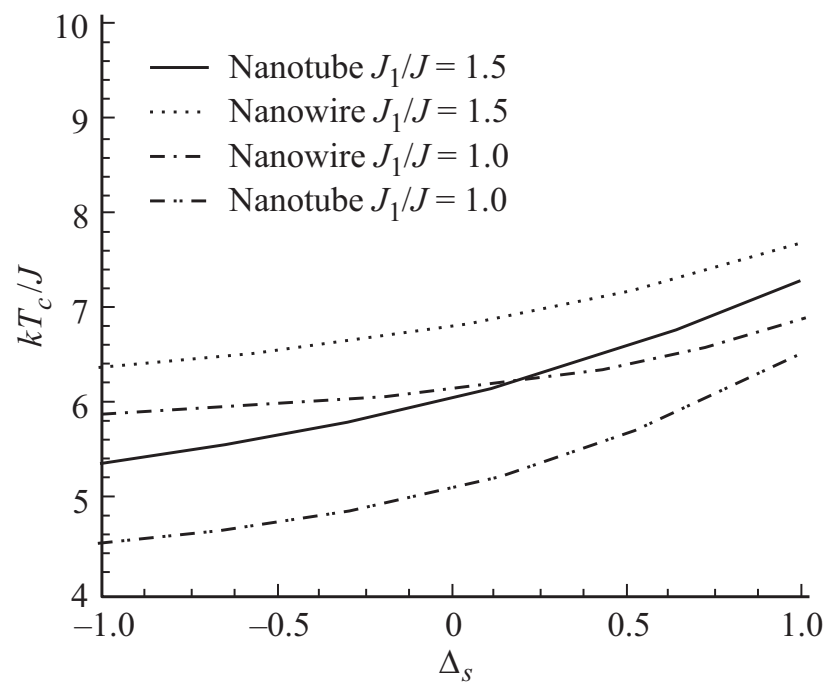

Рис. 2. Критическая температура, как функция от $\Delta_{s}$ для спинов $S_{c}=\frac{1}{2}$ и $S_{s}=1$.

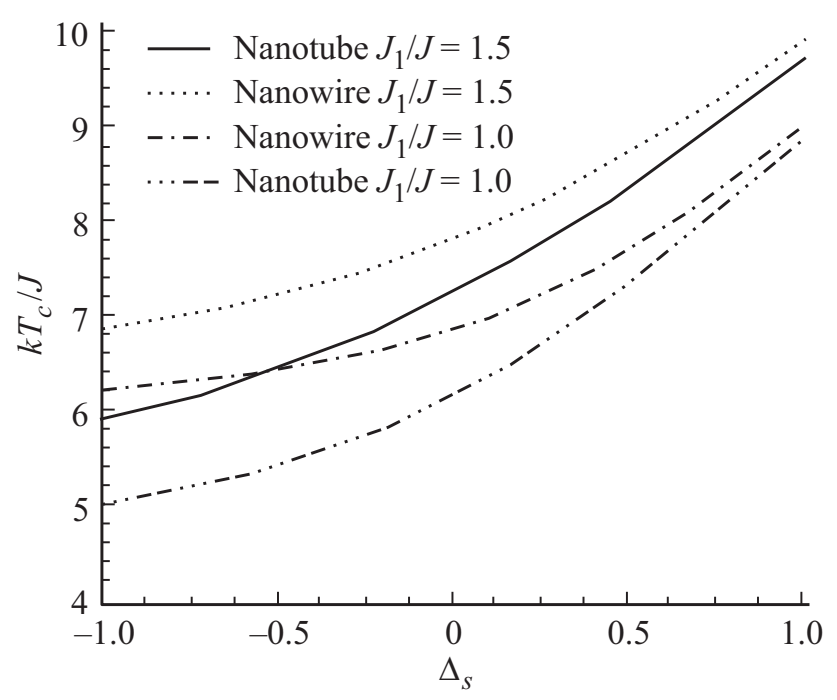

Рис. 3. Критическая температура, как функция от $\Delta_{s}$ для спинов $S_{c}=\frac{1}{2}$ и $S_{s}=\frac{1}{2}$.

В случае, когда связь между сердцевиной и поверхностной стенкой является ферромагнитной, то $J_{1}>0$ и наоборот, если $J_{1}<0$, то данная связь является антиферромагнитной. Для последующего обсуждения определим безразмерный параметр $J_{1} / J$. В дальнейшем фазовые диаграммы будут строиться для случая $J_{1} / J>0$. Также необходимо отметить, что эти диаграммы не изменяются, даже когда знак у $J_{1} / J$ становится отрицательным. Здесь следует заметить, что значение температуры перехода, полученные из уравнения $\operatorname{det} A=0$, не меняется при изменении знака $J_{1} / J$. В порядке обсуждения влияния поверхностных эффектов на температуру перехода (в единицах $J / k)$, показана фазовая диаграмма, как функция $\Delta_{s}$, приведенная на рис. 2 и 3 , для двух положи- тельных значений $J_{1} / J$. Рост $\Delta_{s}$ указывает на увеличение значения отношения поверхностного обмена $J_{s}$ к обмену во внутренней стенке $J$.

На рис. 2 и 3 проиллюстрированы случаи с различными спиновыми моментами $\left(S_{c}=\frac{1}{2} ; S_{s}=1\right)$ и $\left(S_{c}=\frac{1}{2}\right.$; $\left.S_{s}=\frac{1}{2}\right)$ соответственно. Для этого случая были выбраны величины обменных констант, такие как $\left(J_{1} / J=1\right.$ и $\left.J_{1} / J=1.5\right)$. При этом спины, находящиеся в сердцевине и на поверхностной стенке направлены параллельно друг другу и система проявляет ферромагнитную конфигурацию. Сравнивая эти диаграммы двух рассматриваемых наносистем, можно заметить, что фазовая диаграмма нанотрубки схожа с фазовой диаграммой нанопроволоки. Разница между критическими температурами нанопроволоки по сравнению с нанотрубкой увеличивается с уменьшением относительного параметра $J_{1} / J$.

Анализ показывает, что критическая температура увеличивается с увеличением величины $\Delta_{s}$, то есть роль спинового обмена поверхностных спинов в фазовом переходе является весьма существенной. Благодаря тому, что спины сердцевины нанопроволоки имеют большее магнитное упорядочение, чем те же, что в нанотрубке, температура перехода для нанотрубок ниже, чем в нанопроволоках. С другой стороны, как показано на pис. 3 , температура перехода для группы спинов $\left(S_{c}=\frac{1}{2}\right.$; $\left.S_{s}=\frac{1}{2}\right)$ выше, чем для группы $\left(S_{c}=\frac{1}{2} ; S_{s}=1\right)$, приведенных на рис. 2 , что также указывает на определяющую роль поверхностных спинов.

\section{4. Заключение}

Используемый в настойщей работе метод можно считать довольно простым, но удобным для теоретического исследования различного рода магнитных нанообъектов, сверхрешеток и других нестандартных магнитных структур. В настоящей работе была рассчитана температура перехода в зависимости от различного типа обменных констант и спиновых моментов. Было показано, что с ростом поверхностного обмена температура перехода значительно возрастает и сравнительно выше в нанопроволоках чем в нанотрубках,. Полученные результаты позволяют произвести качественную и количественную оценку поведения температуры фазового перехода в широком интервале спиновых и обменных параметров. Подобная модель нанопроволок и нанотрубок может быть применена и к вышеуказанным, во введении, нанообъектам с учетом их специфических структурных и физических особенностей.

\section{Список литературы}

[1] S. Castillo-Sepulveda, R.M. Corona, D. Altbir, J. Escrig. J. Magn. Magn. Mater. 416, 325 (2016).

[2] D. Neumaier, D. Vogl, J. Eroms, D. Weiss. Phys. Rev. B 78, 174424 (2008). 
[3] N. Gao, H.J. Wang, E.H. Yang. Nanotechnology 21, 105107 (2010).

[4] V.S. Tkachenko, V.V. Kruglyak, A.N. Kuchko. Phys. Rev. B 81, 024425 (2010).

[5] E.A. Rando, S. Allende. J. Appl.Phys. 118, 013905 (2015).

[6] A.L. Gonzalez, P. Landeros, A.S. Nunez. J. Magn. Magn. Mater. 322, 530 (2010).

[7] Q.A. Pankhurst, J. Connolly, S.K. Jones, J. Dobson. J. Phys. D 36, R167-R181 (2003).

[8] Pedro Tartaj, Marıa del Puerto Morales, Sabino VeintemillasVerdaguer, Teresita Gonzalez-Carreno, Carlos J Serna. J. Phys. D 36, R182 (2003).

[9] Z.K. Wang, M.H. Kuok, S.C. Ng, D.J. Lockwood, M.G. Cottam, K. Nielsch, R.B. Wehrspohn, U. Gösele. Phys. Rev. Lett. 89, 2, 027201 (2002).

[10] O.A. Tretiakov, Ar. Abanov. Phys. Rev. Lett. 105, 157201 (2010).

[11] A. Ghaddar, F. Gloaguen, J. Gieraltowski. J. Phys. Conf. Ser. 200, 072032 (2010).

[12] E.L. Bizdioca, M. Spasova, M. Farle, M. Hilgendorff, F.J. Caruso. J. Magn. Magn. Mater. 240, 44 (2002).

[13] M. Droz, A. Maritan, A.L. Stella. Phys. Lett. A 92, 287 (1982).

[14] T. Kaneyoshi. Phys. Status Solidi B 248 1, 250 (2011).

[15] X. Lin, G. Ju, T. Gao, X.Chang, Y. Liu, H. Zhang, Y. Du. Solid State Commun. 151, 1708 (2011).

[16] W. Chen, Z.Li, G. B.Ji, S.L. Tang, M. Lu, Y.W. Du. Solid State Commun. 133, 235 (2005).

[17] C. Rousse, P. Fricoteaux. J. Mater. Sci. 46, 6046 (2011).

[18] L. Zhang, Y. Zhang. J. Magn. Magn. Mater. 321, L15 (2009).

[19] J.P. Xu, Z.Z. Zhang, B. Ma, Q.Y. Jin. J. Appl. Phys. 109, $07 \mathrm{~B} 704$.

[20] Y.C. Su, R. Scomski, K.D. Sorge, D.J. Sellmyer. Appl. Phys. Lett. 84, 1525 (2004).

[21] F. Michael, C. Gonzales, V. Mujica, M. Marques, M.A. Ratner. Phys. Rev. B 76224409 (2007).

[22] V.A. Tanriverdiyev. J. Magn. Magn. Mater. 393, 188 (2015).

[23] M. Keskin, N. Sarli, B. Deviren. Solid State Commun. 151, 1025 (2011).

[24] M. Vasilakaki, K.N. Trohidou. Phys. Rev. B 79, 144402 (2009).

Редактор Д.В. Жуманов 